\title{
Video-image processing applied to the analysis of the behaviour of deep-water lobsters (Nephrops norvegicus)
}

\author{
Sergi Pons \\ and Jaume Piera \\ Marinte Technology Unit (UTM-CSIC) \\ Centre Mediterrani d'Investigacions Marines i Ambientals \\ Passeig Martim de la Barceloneta, 37-49 \\ E-08003 Barcelona (Spain) \\ \{spons, jpiera\}@utm.csic.es
}

\author{
Jacopo Aguzzi \\ Institute of Marine Sciences (ICM-CSIC) \\ Centre Mediterrani d'Investigacions Marines i Ambientals \\ Passeig Martim de la Barceloneta, 37-49 \\ E-08003 Barcelona (Spain) \\ jaguzzi@icm.csic.es
}

\begin{abstract}
In this study, we show the results of applying two automated data processing methods to a set of videos previously used to manually monitor the period and the phase of activity rhythms of the deep-water Norway lobster, Nephrops norvegicus, a species of elevated commercial fishery value. Present data are consistent with published findings on Nephrops norvegicus activity rhythms both in the laboratory and the field
\end{abstract}

\section{INTRODUCTION}

Behavioural rhythms are the product of neural mechanism (i.e., the biological clock) that regulates behaviour and internal physiology of animals in relation to those geophysical cycles produced by the Earth during the rotation on its own axis and around the sun (e.g., light intensity and tidal cycles) (reviewed by [1]). In fact, the expression of rhythmic behaviour can be considered as the phenotype of biological clocks regulation (reviewed by [2]). Biological clocks drive the internal biology of organisms and the overt change in activity rate strictly depends and hence coincides, with their mode of functioning. In this sense, neuroethology studies behaviour to understand the neuronal regulation and video-image analysis can be a very efficient tool [3].

Activity rhythms of important fishery resources are to date of interest since affecting their catchability at a diel and seasonal base [4]. The laboratory study of activity rhythms in deep water species is to date limited by technical constrains related to the peculiar animals' environment [5]. A widely adopted methodological solution has been to date the use of infrared (IR) actography (reviewed by [3]). Anyway, highly corrosive salt water impairs the correct functioning of hardware equipment made by IR emitting and detecting barriers. In this context, video image analysis represents a more simple hardware solution when a proper level of automation in frame processing is acquired (reviewed by [6]).

The traditional method of turning data into knowledge relies on the manual analysis and interpretation [7], [8]. For longterm monitoring applications, this form of proceeding is inefficient and expensive. When data volumes grow dramatically manual analyses become completely impractical in many domains. Hence, analysis requires automation [9]. Video-image analysis can be efficiently used to disclose the period and the phase of activity rhythms in relation to the day-night cycle over long period of time in laboratory controlled conditions [10]. The video-image analysis of footages depicting the behavioural pattern of species in relation to time is to date of growing interest for neuroethology and biomedicine [11] but not yet fully exploited for the large augment of frames it produces (from several-days experiments). In this study, we show the results of applying automated data processing to a set of videos previously used to manually monitor the period and the phase of activity rhythms of the deep-water Norway lobster, Nephrops norvegicus, a species of elevated commercial fishery value. These data consist on video images taken from above of clusters of tanks, where each specimen remains isolated from the others.

\section{MATERIALS AND METHODS}

\section{A. The footages}

In this work long lasting footages depicting animals in individualized tanks were used (see [10] for methodological details). Briefly, time lapse video recording were carried out under IR light placing a video camera 2 meters above a group of three poliacrylate individual tanks. Footages were digitized by means of Pinnacle Studio application, obtaining files with DVSD codec, AVI container and a resolution of 720 x 576 pixels (Fig. 1). Then footages where subjected to a pre-processing of gaussian smoothing to reduce the noise level, at expenses of reducing the sharpness of the image. This trade-off resulted on an decrement of false positives when quantifying the amount of motion between consecutive frames. Next, each video stream was splitted according to the tanks sizes, obtaining three new video streams per original video, in order to analyse each individual separately. 


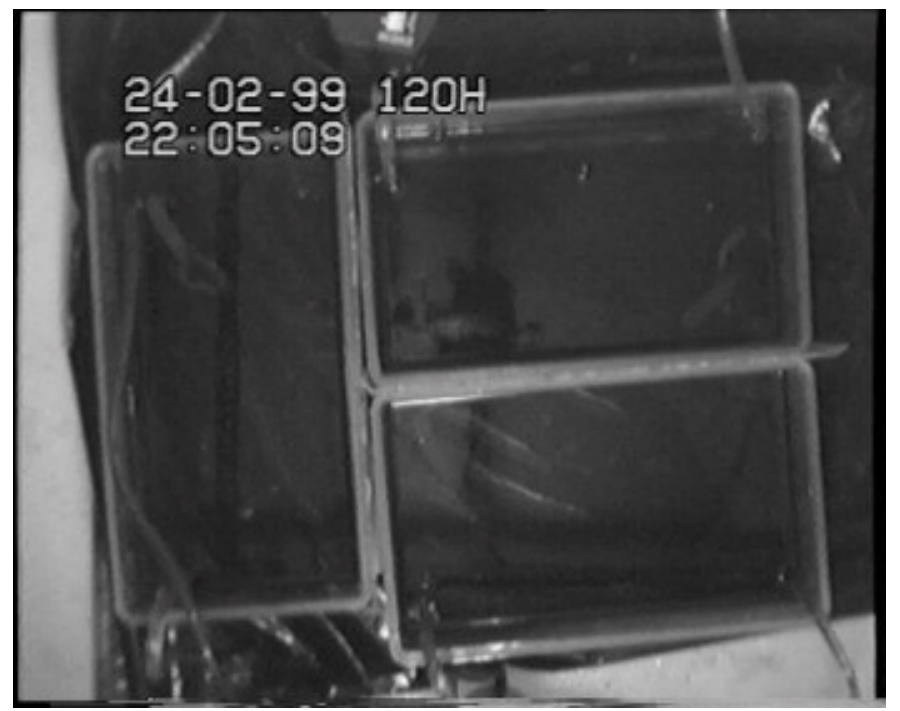

Fig. 1. Sample frame of the video tapes after digitalization

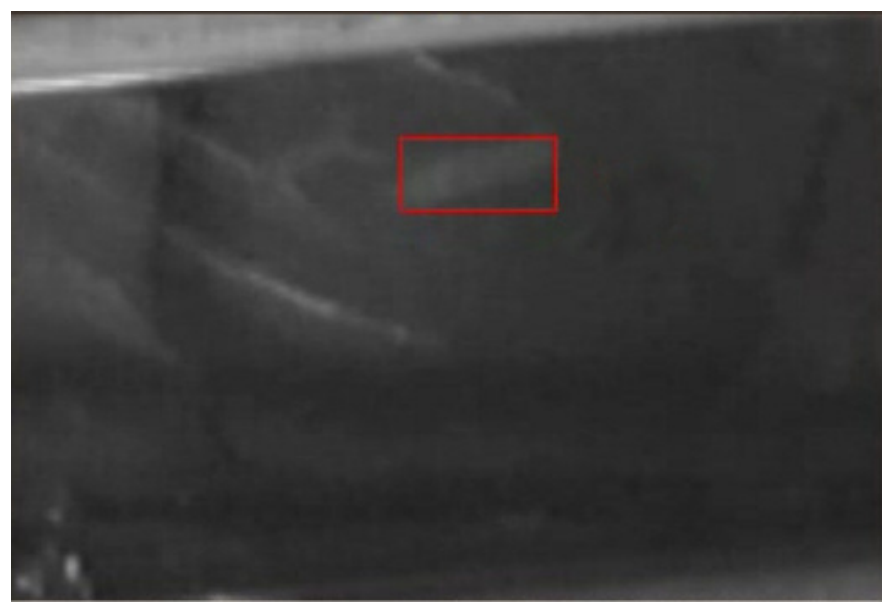

Fig. 2. Original image with bounding box around the lobster.

\section{B. Method 1: Motion estimation from background subtraction}

The process of quantifying motion can be divided into two steps:

1) Separate the foreground object from the background.

2) Measure the displacement of the foreground object between consecutive frames.

The first step can be accomplished obtaining a model of the background, with no foreground objects present, and detecting any changes on it as an indicator of presence. In the case of static scenes, e.g., an empty room under the effect of invariable light conditions, a single frame is enough to create the model. In our data, the following effects forces the use of more complex techniques:

- Bubbles produced by the water refreshing circuit.

- Reflections of artificial light sources on the waves of the water surface.

- Snow noise due to the quality of the VHS tapes.

- Non-uniform illumination, i.e., areas of low contrast.
To overcome these issues, a technique known as codebook background subtraction [12], [13], which creates dynamic background models, has been implemented. Codebook background subtraction works at a pixel level. When the values of a pixel fluctuate between a defined margin for a set period of time, a codebook is created around these values. Any future pixel value inside this codebook is considered background, and the rest foreground. If the new values of the pixel are repeated along the set time instead of the ones of the codebook, the old codebook is deleted and a new one is created. Therefore, the whole set of codebooks of all the pixels represent a compressed form of background model for a long image sequence, and allows us to capture structural background variation due to periodic-like motion over a long period of time under limited memory.

After applying this technique, each frame is transformed to a binarized image with black background and a white foreground object. In order to eliminate non interesting objects, such as bubbles or waves, the image is subjected to a process of erosion. This should completely eliminate the small objects (the undesired phenomena) and shrink the bigger ones (the lobster). Then a dilation process is applied to restore its original size to the remaining objects, which in an ideal case should be only the object representing the lobster. Next step consists on comparing two consecutive black-and-white images, resulting on a relative quantification of the amount of movement. The more than the lobster move, the less than the two foreground objects are superposed and the bigger the difference.

\section{Method 2: Motion estimation from background subtraction and contour finding}

In this method, the same background subtraction technique based on codebooks has been applied. The difference remains on the step of determining the amount of movement. On each binarized frame, cleaned after erosion-dilation process, all the objects are detected with a contour finding algorithm, where a contour is defined by the edge between a black zone and a white zone. The object with the biggest contour is the lobster. The amorphous contour of the lobster is simplified to a bounding box (see Fig. 2), and the center of the box is calculated, assuming that it is approximately the center of the lobster. Finally, the motion is estimated measuring the difference between centers of consecutive images.

\section{RESULTS}

On the original manual analysis of the videos, the parameter measured was how many times the individual crossed from one half of the tank to the other half, in periods of 30 minutes. Although it has provided some worthy results, part of the information about the behaviour is lost (e.g., if the lobster moves without crossing the middle of the tank, it is not taken into account).

On the other hand, the new methods applied allow a precision at frame level. In addition to that, they quantify every single movement of the individual. To ease the comparison of 


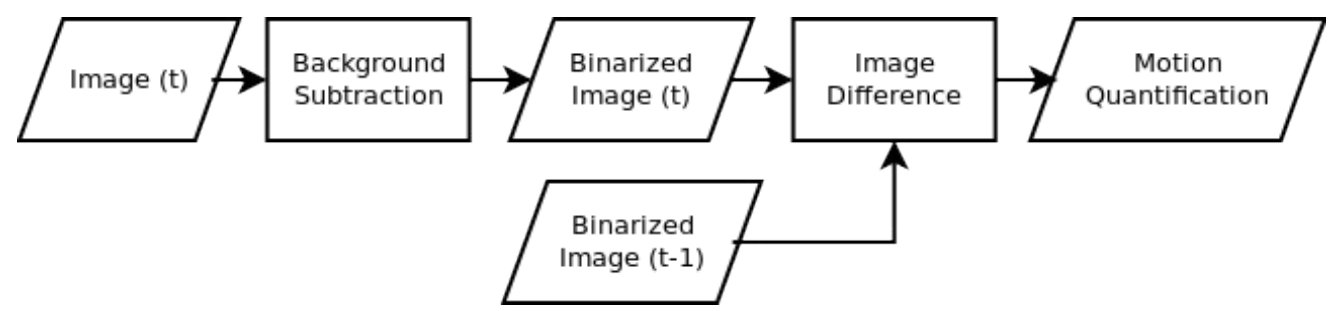

Fig. 3. Work flow of method 1.



Fig. 4. Work flow of method 2.

new data with original data, the new data has been rebinned to periods of 30 minutes. Fig. 5 top shows the data series for a sample tank. Because of the great sensitivity of the methods, a base noise-level can be appreciated. Fig. 5 bottom shows the same data with the base level removed and normalized data ranges to compare it easier to original data.

Fast Fourier Transform (FFT) [14] has been used to detect the presence of periodic events. Fig 6 shows the power spectra from the sample data of Fig. 5. As can be seen, for each data series a relevant frequency is detected. If this frequency is converted to time, original data shows that it contains a periodic signal with a periodicity of approximately 18 hours, data from method 1 shows a periodicity of approximately 21 hours, and data from method 2 shows a periodicity of approximately 25 hours. The Nephrops norvegicus follows a daily activity rhythm, i.e., a periodicity of 24 hours, hence the automatic methods provide results closer to that value.

\section{Discussion}

Present data are consistent with published findings on Nephrops norvegicus activity rhythms both in the laboratory and the field (reviewed by [15]). Animals present marked diel variations in the rate of locomotion in relation to light intensity cycles of different intensity at different depths. In the laboratory, animals show nocturnal activity peaks when light is strong. The observation that automated video image analysis proposes behavioural patterns similar to what observed by [10]) indicates the viability of the used automated protocol.

In the present application we obtained much fine-grained results than on previous studies using manual analysis. Even more, it has been shown that more accurate information can be extracted. This higher frequency sampling, at a frame level, opens the door to a further analysis of the data, to infer new characteristics of the behaviour of the Nephrops norvegicus.

In some videos, significant differences between the results of the two methods has been detected. This is due to the fact that method 1 is more sensible to effects that are not
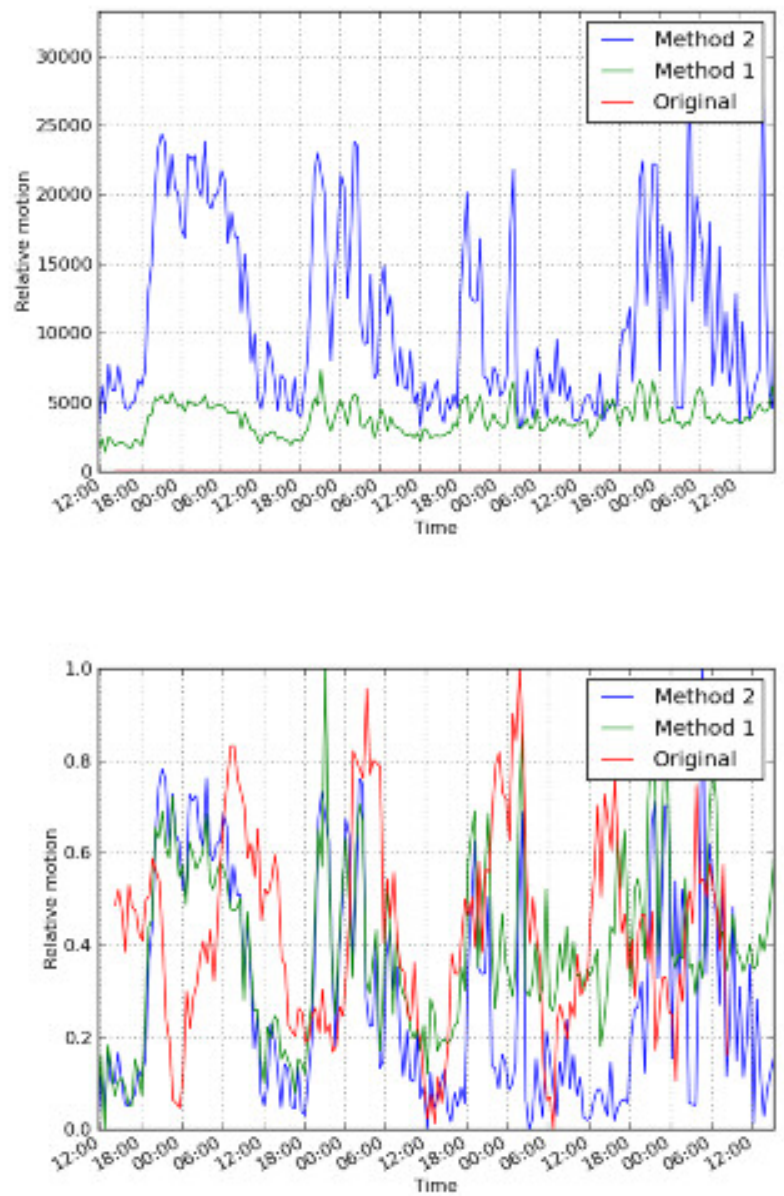

Fig. 5. Comparison of the obtained temporal series with the manual analysis, labelled as original, and the proposed methods, for a given tank. The differences in magnitude can be seen on the top, while the bottom version shows a normalized version of the data to ease its comparison in time. 




Fig. 6. Power spectra from time series data of Fig. 5. The power values have been normalized for comparison purpose. Frequencies proving the daily periodicity are highlighted.

eliminated by the erosion-dilation process. On the other hand, the approximation of the amorphous contour of the lobster to a bounding box can lead to inefficient results when the individual rotates over himself. Hence, an improvement of method 2 would be a better estimation of the center, changing the characteristics of the bounding box.

All the code was developed in $\mathrm{C}++$ using the open source library OpenCV [13], so it can be implemented easily on embedded systems. The posterior analysis of obtained data was performed with Python [16] and SciPy [17], and could be also ported to systems like PC-104 architecture with no effort.

There is a growing interest in the development of lessinvasive and automated techniques for the long-lasting monitoring of behavioural activity in marine organisms [18]. The implementation of automated protocols for the processing of large augment of video information is to date a challenging topic for the exploration of the sea. Automated object tracking and object classification developed in the laboratory represent important steps to investigate poorly accessible environments.

\section{REFERENCES}

[1] J. Dunlap, J. Loros, P. Decoursey et al., Chronobiology: biological timekeeping. Sinauer Associates, 2004.

[2] R. Reffinetti, Circadian physiology. CRC Press, 2005.

[3] J. Aguzzi, D. Sarriá, J. García, J. Del Rio, F. Sardà, and A. Manuel, “A new tracking system for the measurement of diel locomotor rhythms in the Norway lobster, Nephrops norvegicus (L.)," Journal of Neuroscience Methods, vol. 173, no. 2, pp. 215-224, 2008.

[4] E. Naylor, "Chronobiology: implications for marine resource exploitation and management," Scientia Marina, vol. 69, no. S1, 2005.

[5] P. Menesatti, J. Aguzzi, C. Costa, J. García, and F. Sardà, "A new morphometric implemented video-image analysis protocol for the study of social modulation in activity rhythms of marine organisms," Journal of Neuroscience Methods, vol. 184, no. 1, pp. 161-168, 2009.

[6] J. Aguzzi, C. Costa, Y. Fujiwara, R. Iwase, E. Ramirez-Llorda, and P. Menesatti, "A Novel Morphometry-Based Protocol of Automated Video-Image Analysis for Species Recognition and Activity Rhythms Monitoring in Deep-Sea Fauna," Sensors, vol. 9, no. 11, pp. 8438-8455, 2009.
[7] Detection and tracking of objects in underwater video. Washington, D.C: IEEE International Conference on Computer Vision and Pattern Recognition (CVPR), 2004.

[8] An Automated Visual Event Detection System for Cabled Observatory Video. Madeira Portugal: 3rd International Conference on Computer Vision Theory and Applications, January 2008.

[9] U. Fayyad, G. Piatetsky-Shapiro, , and P. Smyth, "From data mining to knowledge discovery in databases," AI Magazine, vol. 17, pp. 37-54, 1996.

[10] J. Aguzzi, J. Company, and P. Abello, "Locomotor activity of continental slope Nephrops norvegicus (decapoda: Nephropidae)," Journal of Crustacean Biology, vol. 24, no. 2, pp. 282-290, 2004.

[11] J. Aguzzi, C. Costa, P. Menesatti, J. García, and F. Sardà, "Monochromatic blue light entrains diel activity cycles in the norway lobster, Nephrops norvegicus (1.) as measured by automated video-image analysis," Scientia Marina, 2009.

[12] K. Kim, T. Chalidabhongse, D. Harwood, and L. Davis, "Real-time foreground-background segmentation using codebook model," RealTime Imaging, vol. 11, no. 3, pp. 172-185, 2005.

[13] G. Bradski and A. Kaehler, Learning OpenCV: Computer vision with the OpenCV library. O'Reilly Media, Inc., 2008.

[14] E. Brigham, The fast Fourier transform and its applications. Prentice Hall Englewood Cliffs, NJ, 1988.

[15] J. Aguzzi and F. Sardà, "A history of recent advancements on Nephrops norvegicus behavioral and physiological rhythms," Reviews in Fish Biology and Fisheries, vol. 18, no. 2, pp. 235-248, 2008.

[16] M. Lutz, Programming python. O'Reilly Media, Inc., 2006.

[17] E. Jones, T. Oliphant, P. Peterson et al., "SciPy: Open source scientific tools for Python," 2001-. [Online]. Available: http://www.scipy.org/

[18] J. Aguzzi and J. Company, "Chronobiology of deep-water decapod crustaceans on continental margins," Advances in Marine Biology, An Annual Review, 2010, accepted. 